\title{
Further Results on High-Rate MIMO-OFDM Underwater Acoustic Communications
}

\author{
Baosheng Li ${ }^{1}$, Jie Huang ${ }^{1}$, Shengli Zhou ${ }^{1}$, Keenan Ball ${ }^{2}$, Milica Stojanovic ${ }^{3}$, Lee Freitag ${ }^{2}$, Peter Willett ${ }^{1}$ \\ ${ }^{1}$ Dept. of Elec. and Computer Engr., University of Connecticut, Storrs, CT 06269 \\ ${ }^{2}$ Woods Hole Oceanographic Institution, Woods Hole, MA 02543 \\ ${ }^{3}$ Massachusetts Institute of Technology, Cambridge, MA 02139
}

\begin{abstract}
MIMO-OFDM is one viable solution for high data rate transmissions over underwater acoustic channels. Our previous work in [1] has reported experimental results for MIMOOFDM with two transmitters and QPSK modulation. In this paper, we deal with MIMO-OFDM with more transmitters and higher order modulation. With data collected from the RACE experiment at Narragansett Bay, Rhode Island, March 2008, we report MIMO-OFDM performance results of QPSK/8QAM/16-QAM/64-QAM modulations with two transmitters, QPSK/8-QAM/16-QAM modulations with three transmitters, and QPSK/8-QAM modulations with four transmitters. With large constellations and MIMO spatial multiplexing, very high data rates can be achieved, e.g., a spectral efficiency of $3.5 \mathrm{bits} / \mathrm{sec} / \mathrm{Hz}$ is approached in three different configurations.
\end{abstract}

\section{INTRODUCTION}

Multicarrier modulation in the form of OFDM has been actively studied for underwater acoustic (UWA) communications [2]-[5]. Also, multi-input and multi-output (MIMO) techniques have been applied to UWA communications that can considerably increase the data rate [6], [7]. MIMOOFDM is one viable solution for very-high-rate transmission by combining the concepts of MIMO and OFDM. In [1], we first presented experimental results for a MIMO-OFDM system with two transmitters and QPSK modulation.

In this paper, we deal with MIMO-OFDM systems with more than two transmitters, and with high order modulation. We adopt an iterative receiver structure that couples a MIMO detector with a channel decoder. The proposed MIMO detector consists of a hybrid use of successive interference cancellation and MMSE equalization with a prior information [8].

With data collected from the RACE experiment at Narragansett Bay, Rhode Island, March 2008, we report performance results of QPSK/8-QAM/16-QAM/64-QAM modulations with two transmitters, QPSK/8-QAM/16-QAM modulations with three transmitters, and QPSK/8-QAM modulations with four transmitters. With large constellations and MIMO spatial multiplexing, very high data rates can be achieved. For example, a spectral efficiency of $3.5 \mathrm{bits} / \mathrm{sec} / \mathrm{Hz}$ is approached in this experiment with two parallel 64-QAM data streams, or three parallel 16-QAM data streams, or four parallel 8-QAM data streams.

B. Li and S. Zhou are supported by the NSF grant ECCS-0725562 and the ONR YIP grant N00014-07-1-0805. M. Stojanovic is supported by the ONR grants N00014-07-1-0202 and N00014-07-1-0738. K. Ball and L. Freitag are supported by the ONR grant N00014-07-1-0229. J. Huang and P. Willett are supported by the ONR grant N00014-07-1-0429.
The rest of the paper is organized as follows. We describe the transmitter design in Section II and present the receiver algorithms in Section III. Experimental results are collected in Section IV and conclusions are drawn in Section V.

\section{Transmitter Design}

We consider a MIMO-OFDM transmission with spatial multiplexing on $N_{t}$ transmitters, where $N_{t}=2,3$, or 4 will be used in the experiment results. Within each OFDM block, $N_{t}$ independent bit streams are encoded with a low-densityparity-check (LDPC) code [9] separately. The coded bits are mapped into information symbols using QPSK, 8-QAM, 16QAM, or 64 QAM modulations. There are $N_{t}$ OFDM blocks formed from the $N_{t}$ streams of information sequences and transmitted through $N_{t}$ transmitters simultaneously. On each transmitter, we use the zero-padded (ZP) OFDM format as in [5]. The key transmission parameters for experimental results in this paper are listed in Table I.

TABLE I

ZP-OFDM PARAMETERS

\begin{tabular}{|l|l|}
\hline Signal bandwidth & $B=4.8828 \mathrm{kHz}$ \\
\hline OFDM block duration & $T=209.7152 \mathrm{~ms}$ \\
\hline Guard interval & $T_{g}=25 \mathrm{~ms}$ \\
\hline Subcarrier spacing & $\Delta f=4.8 \mathrm{~Hz}$ \\
\hline Number of subcarriers & $K=1024$ \\
\hline Number of data carriers & $K_{d}=672$ \\
\hline Number of pilot carriers & $K_{p}=K / 4=256$ \\
\hline Number of null subcarriers & $K_{n}=96$ \\
\hline
\end{tabular}

Let $B$ denote the bandwidth, $r_{c}$ the code rate, $M$ the constellation size, $T$ the OFDM block duration, $T_{g}$ the guard time, $K$ the total number of subcarriers, and $K_{d}$ the number of data subcarriers. Accounting for all the overheads due to guard interval, channel coding, pilot, and null subcarriers, the overall spectral efficiency in terms of bits per second per $\mathrm{Hz}$ (bits/s/Hz) is:

$$
\alpha=N_{t} \cdot \frac{T}{T+T_{g}} \cdot \frac{K_{d}}{K} \cdot r_{c} \cdot \log _{2} M .
$$

With a bandwidth $B$, the data rate is $R=\alpha B$ bits per second.

\section{ReCEIVER Algorithms}

For stationary MIMO-OFDM tests, no resampling operation as described in [5] is needed. The key processing steps at the receiver are as follows. 


\section{A. Doppler estimation}

We apply the same Doppler estimation algorithm on each receiver as described in [1], [5]. In particular, each receiver assumes one common Doppler shift on all transmitted data streams, and uses the energy on the null subcarriers as an objective function to search for the best Doppler shift estimate.

After Doppler shift estimation and compensation, the average energy on the null subcarriers is used to compute the noise variance, which will be used in the demodulation step.

\section{B. Channel estimation}

We use pilot tones for channel estimation, as described in [1], [5]. When $N_{t}=2$ or $N_{t}=4$, those $K_{p}$ pilot subcarriers are divided into $N_{t}$ non-overlapping groups, with each group containing a set of $K_{p} / N_{t}$ equally-spaced subcarriers. Each group is exclusively used by one transmitter. The signal design for the $N_{t}=3$ case is obtained from that of $N_{t}=4$ by just turning one transmitter off. Hence, the channel estimation step is identical for the $N_{t}=3$ and $N_{t}=4$ settings.

Since $K_{p} / N_{t}$ pilot tones are used for each channel estimator, our transceiver design can handle channels with at most 128 and 64 taps in the baseband discrete-time processing, when $N_{t}=2$ and $N_{t}=4$, respectively. These correspond to channel delay spreads of $26.21 \mathrm{~ms}$ and $13.10 \mathrm{~ms}$ with $B=4.8828 \mathrm{kHz}$.

\section{Iterative MIMO demodulation and decoding}

On each data subcarrier $k$, we stack the data from $N_{r}$ receiving-elements as $\mathbf{y}[k]=\left[y_{1}[k], \ldots, y_{N_{r}}[k]\right]^{T}$. Using the matrix-vector channel model [1], we have

$$
\mathbf{y}[k]=\mathbf{H}[k] \mathbf{s}[k]+\mathbf{w}[k],
$$

where $\mathbf{H}[k]$ is the channel matrix of size $N_{r} \times N_{t}, \mathbf{s}[k]$ contains $N_{t}$ transmitted symbols on the $k$ th subcarrier, and $\mathbf{w}[k]$ is the additive noise. We assume that the noises on different receivers are uncorrelated and Gaussian distributed. The data are properly scaled so that the variances are identical on all receivers.

A maximum a posteriori (MAP) MIMO detector and a linear zero-forcing $(\mathrm{ZF})$ detector were presented in [1] for the setting of two transmitters and QPSK modulation. With large constellation size and large $N_{t}$, the MAP detector is no longer affordable. The ZF detector may suffer from the noise enhancement effect due to matrix inversion. Further, no iteration between MIMO demodulation and channel decoding was used in [1].

Here we adopt the MMSE equalization with a prior information developed in [8] as a component for MIMO demodulation, which is then coupled with the nonbinary LDPC decoding [9] for iterative processing. As LDPC codes can declare decoding success during the decoding process, successive interference cancellation is incorporated to speed up the MIMO demodulation. The iterative MIMO demodulation and decoding steps are as follows.

- Step 1: Initialization. First, we define $N_{t}$ flags to indicate the decoding success of those $N_{t}$ parallel data streams. Initially all flags are set to zero implying no success.
Second, for each symbol in (2) to be demodulated, set the mean to be zero and the variance to be the symbol energy $E_{s}$, i.e., initially each symbol has equal probability in residing on all the constellation points.

Third, to reduce the complexity of MMSE equalization, we project the $N_{r} \times 1$ received vector into the $N_{t}$ dimensional signal space as $N_{r}$ might be much larger than $N_{t}$. (This step is optional though.) Specifically, let $\mathbf{U}[k]$ contains $N_{t}$ basis vectors of the range space of $\mathbf{H}[k]$, which can be found by singular value decomposition. Let $(\cdot)^{\mathcal{H}}$ denote Hermitian transpose. We obtain

$$
\tilde{\mathbf{z}}[k]=\mathbf{U}^{\mathcal{H}}[k] \mathbf{y}[k]=\tilde{\mathbf{A}}[k] \mathbf{s}[k]+\boldsymbol{\xi}[k],
$$

where $\tilde{\mathbf{A}}[k]=\mathbf{U}^{\mathcal{H}}[k] \mathbf{H}[k]$ is now square and $\boldsymbol{\xi}[k]=$ $\mathbf{U}^{\mathcal{H}}[k] \mathbf{w}[k]$ has the same covariance matrix as $\mathbf{w}[k]$.

- Step 2: Interference cancellation. The data streams which are declared with decoding success do not need to be decoded again. Hence, their contributions can be subtracted from the received signals.

Assume that $N$ out of $N_{t}$ data streams remain to be decoded. Partition $\tilde{\mathbf{A}}[k]$ as $\left[\mathbf{A}_{\mathrm{d}}[k], \mathbf{A}_{\mathrm{u}}[k]\right]$, where the first part corresponds to the correctly decoded data streams and the second part corresponds to the streams to be decoded. Similarly for $\mathbf{s}_{\mathrm{d}}[k]$ and $\mathbf{s}_{\mathrm{u}}[k]$. We obtain

$$
\mathbf{z}[k]=\tilde{\mathbf{z}}[k]-\mathbf{A}_{\mathrm{d}}[k] \mathbf{s}_{\mathrm{d}}[k]=\mathbf{A}_{\mathrm{u}}[k] \mathbf{s}_{\mathrm{u}}[k]+\boldsymbol{\xi}[k] .
$$

- Step 3: MMSE equalization with a prior information. On each subcarrier $k$, we run the MMSE equalization algorithm with a prior information from [8]. The inputs to the MMSE equalizer are $\mathbf{z}[k], \mathbf{A}_{\mathrm{u}}[k]$, and the means and variances of all symbols in $\mathbf{s}_{\mathrm{u}}[k]$. The outputs of the MMSE equalizer are the probabilities of each information symbol to be on different constellation points. See the details provided in the Appendix.

- Step 4: Nonbinary LDPC decoding. With the outputs from the MMSE equalizer, non-binary LDPC decoding [9] is run for each data stream to be decoded. The decoder outputs the decoded information symbols and the updated probabilities, which are used to refine the mean and variance of each symbol as

$$
\begin{aligned}
& \bar{s}=\sum_{\alpha_{i} \in \mathcal{S}} \alpha_{i} \cdot P\left(s=\alpha_{i}\right), \\
& v=\left(\sum_{\alpha_{i} \in \mathcal{S}}\left|\alpha_{i}\right|^{2} \cdot P\left(s=\alpha_{i}\right)\right)-|\bar{s}|^{2},
\end{aligned}
$$

where we use $\mathcal{S}=\left\{\alpha_{1}, \alpha_{2}, \ldots, \alpha_{2^{b}}\right\}$ to denote the $2^{b}$-ary modulation alphabet.

During the decoding process, the decoder will declare success if the parity check conditions are satisfied.

- Step 5: Iteration among steps 2, 3, and 4. The iteration will stop after one more round of decoding on the last data stream when other $N_{t}-1$ streams have been successfully decoded as further iteration will not help, or after a number of runs as specified. 


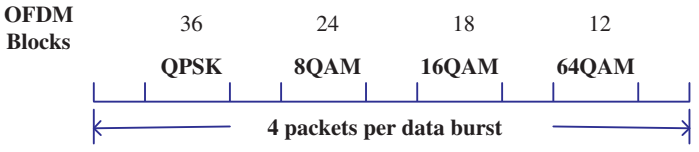

Fig. 1. The structure of the transmission file

\section{EXPERIMENTAL RESULTS}

The Rescheduled Acoustic Communications Experiment (RACE) was held in Narragansett Bay, Rhode Island, March 2008. The water depths in the area range from 9 to about 14 meters. The primary source of an ITC1007 transducer for acoustic transmissions was located approximately 4 meters above the bottom. A vertical source array consisting of three AT-12ET transducers with a spacing of $60 \mathrm{~cm}$ between each transducer was deployed below the primary source. The top of the source array was approximately 1 meter below the primary source. The sampling rate was $f_{s}=39.0625 \mathrm{kHz}$. The center frequency was chosen as $f_{c}=11.5 \mathrm{kHz}$. Other signal parameters are listed in Table I.

Label the four transducers from top to bottom as T0, T1, T2 and T3. For MIMO-OFDM transmissions, we use T0 and T1 for two transmitters, T0-T2 for three transmitters, and T0$\mathrm{T} 3$ for four transmitters. Note that T0 and the T1-T3 array are driven by different power supplies and have different front-end circuits. In addition, driven by the same voltage inputs, the transducer T0 produces less transmission power than T1-T3, about $5 \mathrm{~dB}$ lower comparing the peaks. Finally, the spacing between $\mathrm{T} 0$ and $\mathrm{T} 1$ is larger than the spacings among $\mathrm{T} 1$, T2, T3. Such a disparity between T0 and T1-T3 renders the data stream from $\mathrm{T} 0$ in a disadvantage relative to other data streams; this will be reflected by the performance results.

For each MIMO-OFDM configuration, one data burst consists of four packets with different modulations, as shown in Fig. 1. In particular, the packet of QPSK modulation contains 36 OFDM blocks, the packet of 8-QAM contains 24 OFDM blocks, the packet of 16QAM contains 18 OFDM blocks, while the packet of 64-QAM contains 12 OFDM blocks. (The 8-QAM constellation used in this paper is from [10, Fig. 4.34]). Rate $1 / 2$ nonbinary LDPC coding as described in [9] is applied. Hence, each data burst contains the same number $(672 \times 36=24192)$ of information bits for each parallel data stream at each setting.

Three receiving arrays were deployed during the experiment, mounted on fixed tripods with the bottom of the arrays 2 meters above the sea floor. We here report the results on the array at 400 meters to the east from the source, which is a 24-element vertical array with $5 \mathrm{~cm}$ between elements. We will use the data from the top 12 elements for decoding.

During the experiment, each transmission file was transmitted twice every four hours, leading to 12 transmissions each day. We here report performance results based on data collected from 28 transmissions within the Julian dates 081083. Hence, each data stream at each setting has a total of $28 \times 24192=677,376$ information bits transmitted.

Fig. 2 depicts the channel estimates in one OFDM block

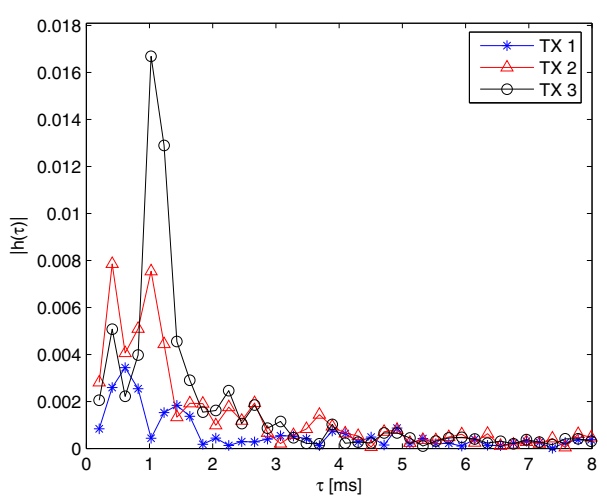

Fig. 2. Channel estimates from one OFDM block with three transmitters.

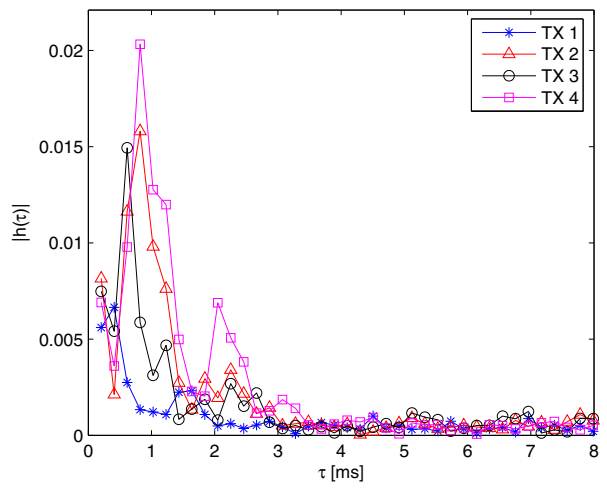

Fig. 3. Channel estimates from one OFDM block with four transmitters.

with three transmitters while Fig. 3 shows the channel estimates in one OFDM block with four transmitters (both from the recorded file 0810156F05_C0_S3). The channel delay spreads are about $5 \mathrm{~ms}$. Note that the channel corresponding to the first data stream (transducer T0) has lower energy than others. This is a general trend for all the received blocks with only a few exceptions.

\section{A. Performance results with two transmitters}

Figs. 4-6 depict the coded block-error-rate (BLER) for each received data set across the Julian dates 081-083. The 8-QAM case is omitted due to zero BLERs across all dates. Decoding errors only occur in one out of 28 data sets in the QPSK case, where two out of 36 OFDM blocks were badly distorted that prevents correct decoding of stream 1. Table II summarizes the coded bit-error-rates (BERs) and BLERs averaged over all data sets; i.e., a total of 677,376 information bits was used for each BER computed in the table.

\section{B. Performance results with three transmitters}

Figs. 7, 8, and 9 depict the coded BLER for each received data set across the Julian dates 081-083. Table III summarizes the BERs and BLERs averaged over all data sets.

\section{Performance results with four transmitters}

Figs. 10 and 11 depict the coded BLER for each received data set across the Julian dates 081-083. Table IV summarizes the BERs and BLERs averaged over all data sets. 


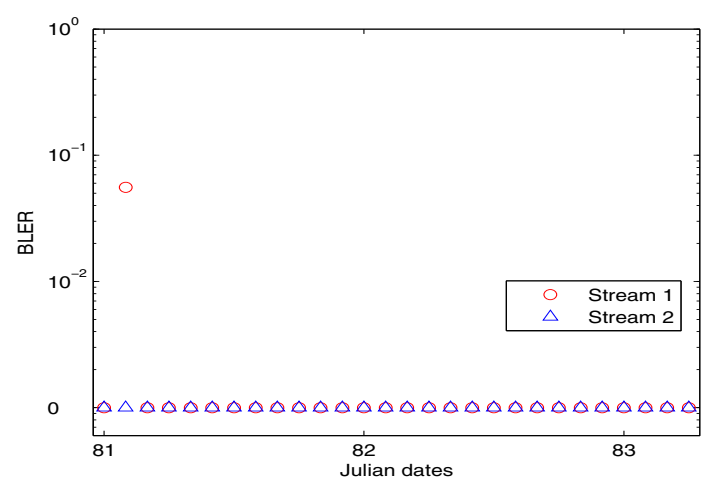

Fig. 4. Block error rates for 2IMO-OFDM, QPSK

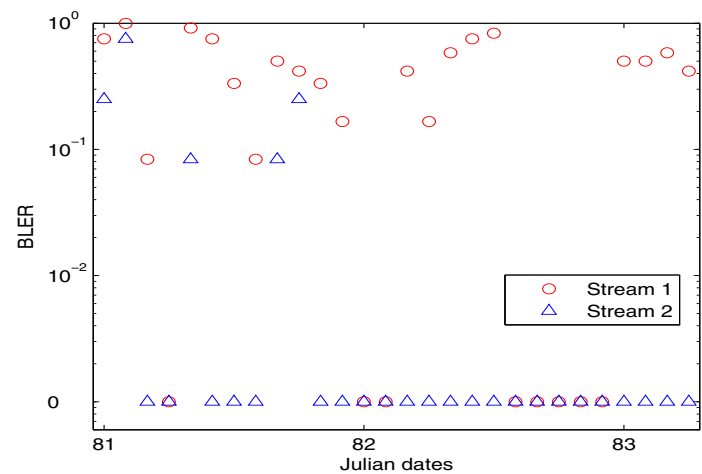

Fig. 6. Block error rates for 2IMO-OFDM, 64-QAM

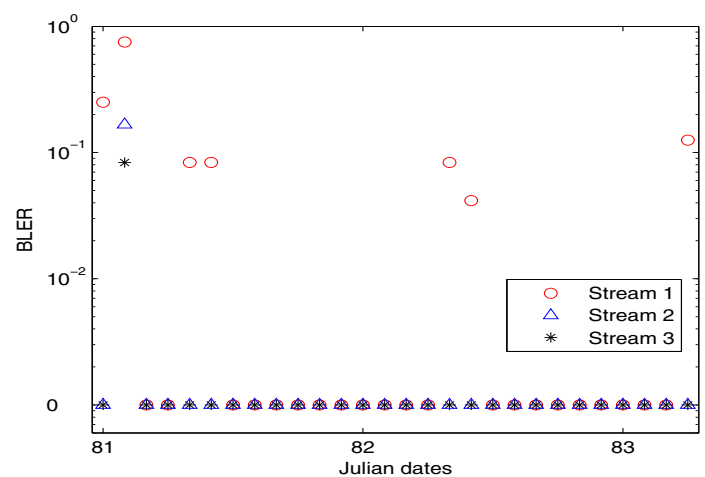

Fig. 8. Block error rates for 3IMO-OFDM, 8-QAM

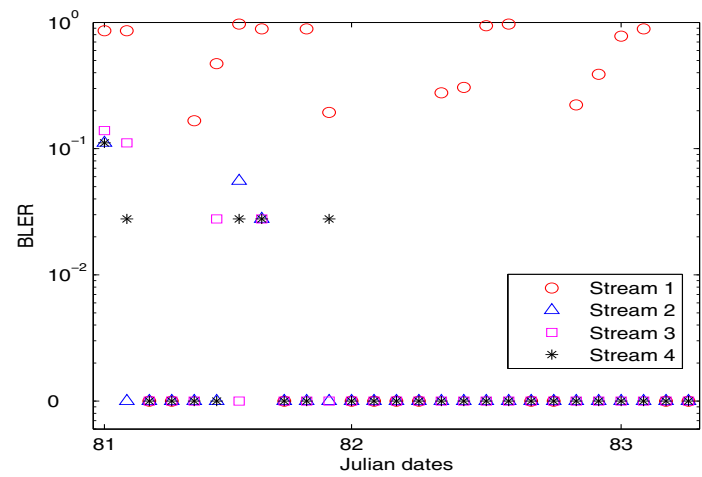

Fig. 10. Block error rates for 4IMO-OFDM, QPSK

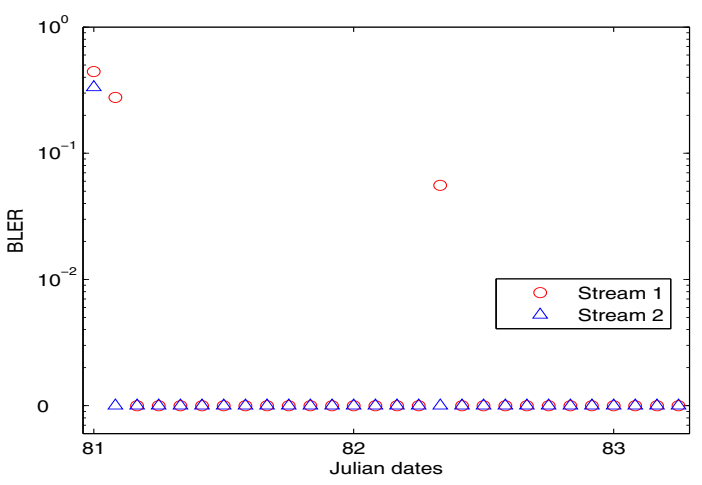

Fig. 5. Block error rates for 2IMO-OFDM, 16-QAM

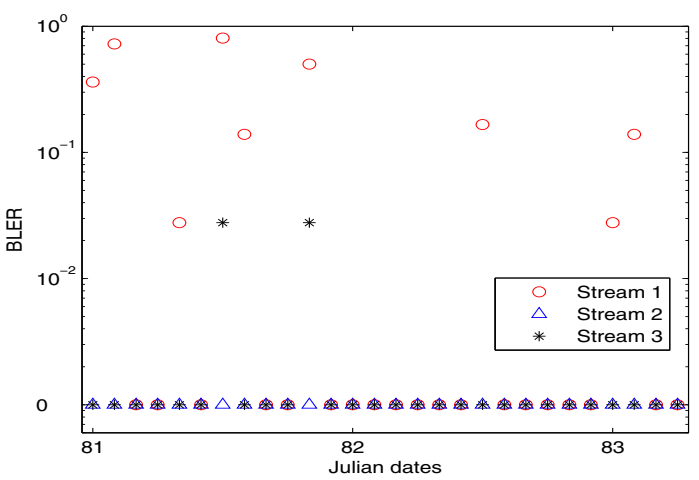

Fig. 7. Block error rates for 3IMO-OFDM, QPSK

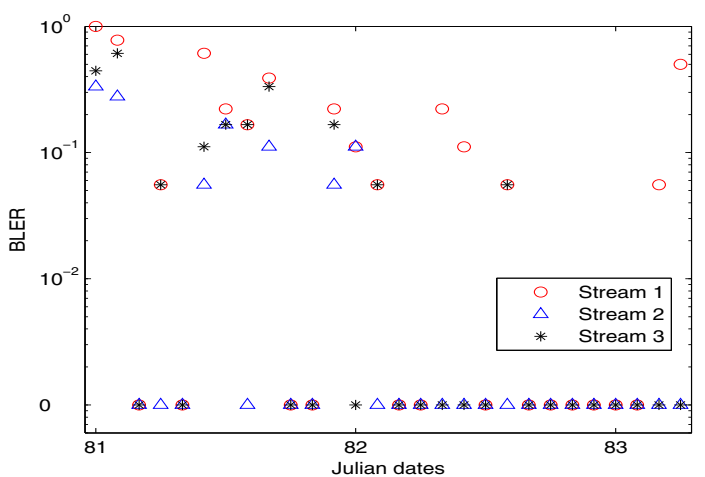

Fig. 9. Block error rates for 3IMO-OFDM, 16-QAM

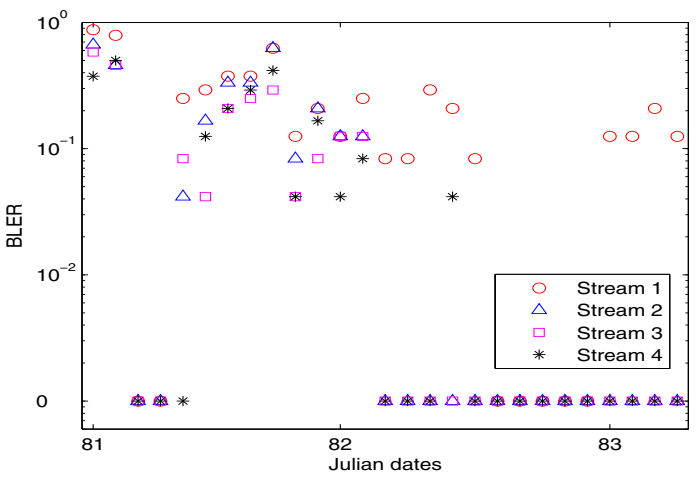

Fig. 11. Block error rates for 4IMO-OFDM, 8-QAM 
TABLE II

Performance Results with Two Transmitters and Twelve Receivers

\begin{tabular}{|c|c|c|c|c|}
\hline & Spectral efficiency & Data streams & Average BER & Average BLER \\
\hline 2IMO, QPSK & 1.17 bits/s/Hz & Stream 1 & $4 \cdot 10^{-4}$ & $2 \cdot 10^{-3}$ \\
& & Stream 2 & 0 & 0 \\
\hline 2IMO, 8-QAM & 1.76 bits/s/Hz & Stream 1 & 0 & 0 \\
& & Stream 2 & 0 & 0 \\
\hline 2IMO, 16-QAM & 2.35 bits/s/Hz & stream 1 & $6 \cdot 10^{-3}$ & $3 \cdot 10^{-2}$ \\
& & stream 2 & $3 \cdot 10^{-3}$ & $1 \cdot 10^{-2}$ \\
\hline 2IMO, 64-QAM & 3.52 bits/s/Hz & Stream 1 & $7 \cdot 10^{-2}$ & $4 \cdot 10^{-1}$ \\
& & Stream 2 & $1 \cdot 10^{-2}$ & $5 \cdot 10^{-2}$ \\
\hline
\end{tabular}

TABLE III

Performance Results with Three Transmitters and Twelve Receivers

\begin{tabular}{|c|c|c|c|c|}
\hline & Spectral efficiency & Data streams & Average BER & Average BLER \\
\hline 3IMO, QPSK & 1.76 bits/s/Hz & Stream 1 & $2 \cdot 10^{-2}$ & $1 \cdot 10^{-1}$ \\
& & Stream 2 & 0 & 0 \\
& & Stream 3 & $5 \cdot 10^{-4}$ & $2 \cdot 10^{-3}$ \\
\hline 3IMO, 8-QAM & 2.64 bits/s/Hz & Stream 1 & $1 \cdot 10^{-2}$ & $5 \cdot 10^{-2}$ \\
& & Stream 2 & $1 \cdot 10^{-3}$ & $6 \cdot 10^{-3}$ \\
& & Stream 3 & $8 \cdot 10^{-4}$ & $3 \cdot 10^{-3}$ \\
\hline 3IMO, 16-QAM & 3.52 bits/s/Hz & Stream 1 & $4 \cdot 10^{-2}$ & $1.6 \cdot 10^{-1}$ \\
& & Stream 2 & $1 \cdot 10^{-2}$ & $4 \cdot 10^{-2}$ \\
& & Stream 3 & $2 \cdot 10^{-2}$ & $8 \cdot 10^{-2}$ \\
\hline
\end{tabular}

TABLE IV

Performance Results with Four Transmitters and Twelve Receivers

\begin{tabular}{|c|c|c|c|c|}
\hline & Spectral efficiency & Data streams & Average BER & Average BLER \\
\hline 4IMO, QPSK & 2.35 bits/s/Hz & Stream 1 & $8 \cdot 10^{-2}$ & $7 \cdot 10^{-2}$ \\
& & Stream 2 & $2 \cdot 10^{-3}$ & $4 \cdot 10^{-2}$ \\
& & Stream 3 & $3 \cdot 10^{-3}$ & $3 \cdot 10^{-2}$ \\
& & Stream 4 & $3 \cdot 10^{-3}$ & $3 \cdot 10^{-2}$ \\
\hline 4IMO, 8-QAM & 3.52 bits/s/Hz & Stream 1 & $4 \cdot 10^{-1}$ & $2 \cdot 10^{-1}$ \\
& & Stream 2 & $7 \cdot 10^{-3}$ & $1 \cdot 10^{-1}$ \\
& & Stream 3 & $1 \cdot 10^{-2}$ & $8 \cdot 10^{-2}$ \\
& & Stream 4 & $8 \cdot 10^{-3}$ & $8 \cdot 10^{-2}$ \\
\hline
\end{tabular}

Usually no more than five iterations are needed between MIMO demodulation and decoding. From Tables II-IV, we observe that data steam 1 has worse performance than other data streams. This is due to the fact that the transducer on T0 has less power efficiency than others, and a possible Doppler shift mismatch between T0 and the array T1-T3 due to different spacings and front-end circuits. The BLER performance for all other data streams except stream 1 are acceptable and actually very good in many cases. A closer look into Figs. 7-11 reveals that no error occurred in the majority of the data sets within the three-day span. The particular case of two transmitters and 8-QAM modulation having a spectral efficiency of $1.76 \mathrm{bits} / \mathrm{s} / \mathrm{Hz}$ does not have any decoding error across all the 28 data sets across three days.

In short, the spectral efficiency can be increased considerably by using high order modulation in MIMO-OFDM transmissions; see various values corresponding to different configurations in Tables II-IV. In particular, a spectral efficiency of $3.52 \mathrm{bits} / \mathrm{sec} / \mathrm{Hz}$ is approached by two parallel 64QAM data streams, or three parallel 16-QAM data streams, or four parallel 8-QAM data streams.

\section{CONCLuSions}

In this paper, we dealt with MIMO-OFDM with more than two transmitters and high order constellations. We presented an iterative receiver that couples MIMO demodulation with nonbinary LDPC decoding, where the MIMO detector consists of a hybrid use of successive interference cancellation and MMSE equalization with a prior information. Based on the data from the RACE08 experiment, we reported MIMOOFDM performance results of QPSK/8-QAM/16-QAM/64QAM modulations with two transmitters, QPSK/8-QAM/16QAM modulations with three transmitters, and QPSK/8-QAM modulations with four transmitters.

\section{APPENDIX: MMSE EQUALIZATION WITH A PRIOR INFORMATION [8]}

For convenience, we list here the MMSE equalization algorithm with a prior information from [8].

We omit the index $k$ and the subscript in (4) to work on a generic model $\mathbf{z}=\mathbf{A} \mathbf{s}+\boldsymbol{\xi}$, where $\boldsymbol{\xi}$ has a covariance matrix $\sigma_{w}^{2} \mathbf{I}_{N}$. The a priori information of $s_{n}, n=1,2, \ldots, N$, is given in the forms of the mean $\bar{s}_{n} \triangleq \mathrm{E}\left(s_{n}\right)$ and the variance 
$v_{n} \triangleq \operatorname{Cov}\left(s_{n}, s_{n}\right)$. Let $\mathbf{a}_{n}$ denote the $n$-th column of matrix A. And use $\bar{s}_{n}$ and $v_{n}$ to define:

$$
\begin{aligned}
\overline{\mathbf{s}} & \triangleq \mathrm{E}(\mathbf{s})=\left[\bar{s}_{1}, \bar{s}_{2}, \ldots, \bar{s}_{N}\right]^{T}, \\
\overline{\mathbf{z}} & \triangleq \mathrm{E}(\mathbf{z})=\mathbf{A E}(\mathbf{s})=\mathbf{A} \overline{\mathbf{s}}, \\
\mathbf{V} & \triangleq \operatorname{Cov}(\mathbf{s}, \mathbf{s})=\operatorname{diag}\left[v_{1}, v_{2}, \ldots, v_{N}\right] \\
\mathbf{\Sigma} & \triangleq \operatorname{Cov}(\mathbf{z}, \mathbf{z})=\sigma_{w}^{2} \mathbf{I}_{N}+\mathbf{A V A} \mathbf{H}^{\mathcal{H}} \\
\mathbf{f}_{n} & \triangleq \mathbf{\Sigma}^{-1} \mathbf{a}_{n}, \\
K_{n} & \triangleq\left(1+\left(1-v_{n}\right) \mathbf{f}_{n}^{\mathcal{H}} \mathbf{a}_{n}\right)^{-1} .
\end{aligned}
$$

The estimate $\hat{s}_{n}$ is then computed as

$$
\hat{s}_{n}=K_{n} \cdot \mathbf{f}_{n}^{\mathcal{H}}\left(\mathbf{z}-\overline{\mathbf{z}}+\bar{s}_{n} \mathbf{a}_{n}\right) .
$$

In this computation, $\hat{s}_{n}$ is independent from the a priori information about $s_{n}$, but dependent on the a prior information about all $s_{n^{\prime}}$ where $n^{\prime} \neq n[8]$.

Assuming that $\hat{s}_{n}$ is Gaussian distributed with mean

$$
\mu_{n}=K_{n} \cdot s_{n} \cdot \mathbf{f}_{n}^{\mathcal{H}} \mathbf{a}_{n}
$$

and variance

$$
\sigma_{n}^{2}=K_{n}^{2} \cdot\left(\mathbf{f}_{n}^{\mathcal{H}} \mathbf{a}_{n}-v_{n} \mathbf{f}_{n}^{\mathcal{H}} \mathbf{a}_{n} \mathbf{a}_{n}^{\mathcal{H}} \mathbf{f}_{n}\right),
$$

the probabilities of $p\left(\hat{s}_{n} \mid s_{n}=\alpha_{i}\right), i=1,2, \ldots, 2^{b}$, can be computed from Gaussian probability density function [8]. The probabilities are passed to the nonbinary LDPC decoder.

\section{ACKNOWLEDGEMENT}

We are grateful to Dr. James Preisig and his team for conducting the RACE08 experiment.

\section{REFERENCES}

[1] B. Li, S. Zhou, M. Stojanovic, L. Freitag, J. Huang, and P. Willett, "MIMO-OFDM over an underwater acoustic channel," in Proc. of MTS/IEEE OCEANS conference, Vancouver, BC, Canada, Sept. 29 Oct. 4, 2007.

[2] M. Chitre, S. H. Ong, and J. Potter, "Performance of coded OFDM in very shallow water channels and snapping shrimp noise," in Proceedings of MTS/IEEE OCEANS, vol. 2, 2005, pp. 996-1001.

[3] P. J. Gendron, "Orthogonal frequency division multiplexing with on-offkeying: Noncoherent performance bounds, receiver design and experimental results," U.S. Navy Journal of Underwater Acoustics, vol. 56, no. 2, pp. 267-300, Apr. 2006.

[4] M. Stojanovic, "Low complexity OFDM detector for underwater channels," in Proc. of MTS/IEEE OCEANS conference, Boston, MA, Sept. 18-21, 2006.

[5] B. Li, S. Zhou, M. Stojanovic, L. Freitag, and P. Willett, "Multicarrier communication over underwater acoustic channels with nonuniform Doppler shifts," IEEE Journal of Oceanic Engineering, vol. 33, no. 2, Apr. 2008.

[6] D. B. Kilfoyle, J. C. Preisig, and A. B. Baggeroer, "Spatial modulation experiments in the underwater acoustic channel," IEEE Journal of Oceanic Engineering, vol. 30, no. 2, pp. 406-415, Apr. 2005.

[7] S. Roy, T. M. Duman, V. McDonald, and J. G. Proakis, "High rate communication for underwater acoustic channels using multiple transmitters and space-time coding: Receiver structures and experimental results," IEEE Journal of Oceanic Engineering, vol. 32, no. 3, pp. 663-688, July 2007.

[8] M. Tuchler, A. C. Singer, and R. Koetter, "Minimum mean squared error equalization using a priori information," IEEE Transactions on Signal Processing, vol. 50, no. 3, pp. 673-683, Mar. 2002.

[9] J. Huang, S. Zhou, and P. Willett, "Nonbinary LDPC coding for multicarrier underwater acoustic communication," in Proc. of MTS/IEEE OCEANS conference, Kobe, Japan, April 8-11, 2008.

[10] J. G. Proakis, Digital Communications. McGraw-Hill,4th edition, 2001. 